\title{
STABILIZATION OF CHAOS WITH FPIC: APPLICATION TO ZAD-STRATEGY BUCK CONVERTERS
}

\author{
Fabiola Angulo* Enric Fossas ${ }^{* *}$ Carlos Ocampo ${ }^{* * *}$ \\ Gerard Olivar ${ }^{* * * *, 1}$ \\ ${ }^{*}$ Department of Electrical, Electronic and Computer \\ Science, Universidad Nacional de Colombia. \\ Cra. 27 No. 64-60, Manizales, Colombia. \\ fangulo@nevado.manizales . unal.edu.co \\ ** Institute of Industrial and Control Engineering, \\ Technical University of Catalonia, Avda. Diagonal 647, \\ pl 11, 08027 Barcelona, Spain. enric.fossas@upc.edu \\ *** Departament of Systems Engineering, Automatics and \\ Industrial Informatics (ESAII), Technical University of \\ Catalonia, Rambla Sant Nebridi, 10, planta 1, Ed TR-11, \\ 08222 Terrasa, Spain. carlos.ocampo@upc.edu \\ **** Department of Applied Mathematics 4, Technical \\ University of Catalonia, Avda. Vctor Balaguer, $s / n$ \\ E-08800 Vilanova i la Geltru, Spain. gerard@mat.upc.edu
}

\begin{abstract}
In this paper a new design for controlling dynamical systems has been applied to buck converters driven with a PWM centered pulse and (Zero Average Dynamics) ZAD-strategy. Chaotic operation has been stabilized with the so-called Fixed Point Induced Control (FPIC) technique. Also, the same converter with delay, whose 1-periodic orbit is always unstable, has been stabilized. Simulations are compared with the TDAS stabilization technique. Once a 1-periodic orbit has been stabilized, the dynamics shows the same characteristics and advantages of the ZAD design, such as robustness, fixed switching frequency (with zero average on the sliding surface) and a low output error. Copyright (c)2005 IFAC
\end{abstract}

Keywords: Chaos, Control Theory, Converters, Nonlinear Control, Stabilization methods.

\section{INTRODUCTION}

Switching sources are devices used in the implementation of power converters. One of its main drawbacks is the presence of chattering due to switching frequency, high order harmonic distortion and nonlinear phenomena. The latter

\footnotetext{
1 This work was supported by the European Union under Grant SICONOS IST 2001-37172.
}

can be dealt with control techniques (Poddar et al., 1998),(Poddar et al., 1995a),(Poddar et al., 1995b),(Batlle et al., 1999),(Batlle et al., 1997),(Batlle et al., 2000) while chattering and harmonic distortion are inherent to switching and can not be totally avoided. Fixed switching frequency improves performance even though chattering does not disappear. This is important in real applications, and to achieve this, some tech- 
niques have been developed: adaptive hysteresis band (Ruiz et al., 1990), (Yao and Holmes, 1993),(Pinheiro et al., 1994), signal injection with a selected frequency (Pinheiro et al., 1994),(Silva and Snia, 1993),(Malesani et al., 1996),(Nicolas et al., 1996), zero average current in each iteration (ZACE) (Borle and Chemmangot, 1995) and recently zero average error dynamics in each iteration (ZAD). ZAD control scheme, recently proposed in (Fossas et al., 2001), tries to conjugate the advantages of fixed frequency implementations and the inherent robustness of sliding control modes. It is based on an appropriate design of the output that guarantees the fulfilment of the specifications and on a specific design of the Pulse Width Modulator duty cycle in such a way that the output average in each PWM-period is zero. A comparative study of this algorithm with respect to some of the previously reported in the literature can be found in (Biel et al., 2002) and (Ramos et al., 2003).

There are several possible PWM implementations, such as leading, trailing and centered pulse. The novel ZAD algorithm implemented in a single updated centered PWM results in a very simple control scheme (CPWM). However, a one period delay in sampling, which one has to consider in real applications makes the closed loop system unstable. This paper deals with a new control methodology named Fixed Point Induction Control (FPIC), that combines the unstabilising control and a nominal control action. The trade off between the two control action weights guarantees system stability keeping the advantages of the ZAD controller. Thus the method is original and very application oriented.

For robustness purposes a linear combination of the error and its derivative is considered as in (Carpita et al., 1988). As it will be seen in the following, the error dynamics time constant appears as a bifurcation parameter. As it varies, a very rich dynamics is observed in the controlled system. A more realistic one-cycle delayed ZAD centered scheme (DCPWM) results in unstable dynamics for all of the $k_{s}$-parameter values. Fig. 1 shows the bifurcation diagram of the normalized dutycycle of a ZAD-CPWM controlled buck converter with delay. It is worth to notice the instantaneous presence of chaos for $k_{s}=5$ due to a bordercollision bifurcation (the duty cycle drops to zero). Two of the authors in (Batlle et al., 1999),(Batlle et al., 1997) applied Time Delayed AutoSynchronisation techniques (TDAS) to stabilize unstable orbits in a ramp-controlled buck converter. These techniques have also been applied to stabilize 1periodic orbits appearing close to the chaotic attractor in the ZAD-CPWM buck converter. However TDAS failed in the ZAD-DCPWM. The proposed FPIC methodology succeeds in both cases.

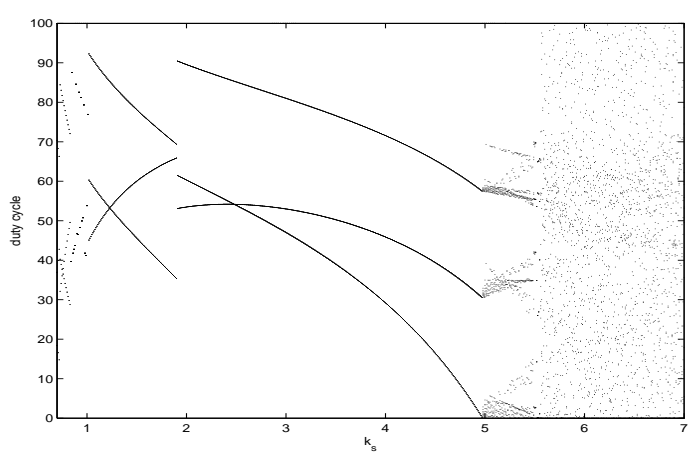

Fig. 1. Bifurcation diagram for the normalized duty cycle in a DCPWM Buck converter when the parameter $k_{s}$ is varied. Instant chaos is present for $k_{s}=5$ after a bordercollision bifurcation (the duty cycle saturates to zero).

The paper is organized as follows: in Section II the theoretical results on FPIC methodology is discussed. In Section III the ZAD strategy is briefly explained. This Section is also devoted to control of chaos in the CPWM system, both with TDAS and FPIC techniques. Section IV deals with the regulation task in a DCPWM, and finally, conclusions are summarized in Section V.

\section{FIXED POINT INDUCTION CONTROL (FPIC)}

Let $\Sigma$ be a discrete system

$$
\mathbf{x}(k+1)=\mathbf{f}(\mathbf{x}(k), u(\mathbf{x}(k)))
$$

where $\mathbf{x} \in \mathbb{R}^{n}$ and $\mathbf{f}: \mathbb{R}^{n+1} \mapsto \mathbb{R}^{n}$. Let $\left(\mathbf{x}^{*}, u^{*}\right)$ be a stable fixed point. Neither assumption is made on the stability of the controlled system, nor on the control law $u(\mathbf{x}(\cdot))$. Particularly we are interested in systems for which the dynamics defined by the map $\mathbf{f}(\mathbf{x}(k), u(\mathbf{x}(k)))$ is stable but $\mathbf{f}(\mathbf{x}(k), u(\mathbf{x}(k-1)))$ becomes unstable.

Result 1: Let $\left(\mathbf{x}^{*}, u^{*}\right)$ be a fixed point of $\mathbf{f}(\mathbf{x}, u)$ and assume that the eigenvalues of the linearised system at the fixed point have modulus less than 1 , i.e.

$$
\left|\lambda_{i}\left(\mathbf{J}_{o}\right)\right|=\left|\lambda_{i}\left(\left.\frac{\partial \mathbf{f}}{\partial \mathbf{x}}\right|_{\left(\mathbf{x}^{*}, u^{*}\right)}\right)\right|<1 \forall i .
$$

Then, the control input (FPIC technique)

$$
\hat{u}(k)=\frac{u(\mathbf{x}(k))+N u^{*}}{N+1}
$$

stabilises the fixed point $\left(\mathbf{x}^{*}, u^{*}\right)$ for $N$ sufficiently high.

Proof:

The equation describing the system with the new controller is

$$
\mathbf{x}(k+1)=\mathbf{f}(\mathbf{x}(k), \hat{u}(k)) .
$$


From Eq. (3) we have that $\left(\mathbf{x}^{*}, u^{*}\right)$ is still a fixed point of the discrete system (4). Linearising system (4) around the fixed point, we have

$$
\mathbf{J}_{n}=\left.\frac{\partial \mathbf{f}}{\partial \mathbf{x}}\right|_{\left(\mathbf{x}^{*}, \hat{u}^{*}\right)}+\frac{\partial \mathbf{f}}{\partial \hat{u}} \frac{\partial \hat{u}}{\partial u} \frac{\partial u}{\partial \mathbf{x}}
$$

that can be written as

$$
\mathbf{J}_{n}=\mathbf{A}_{1}+\mathbf{A}_{2}
$$

with $\mathbf{A}_{1}=\left.\frac{\partial \mathbf{f}}{\partial \mathbf{x}}\right|_{\left(\mathbf{x}^{*}, \hat{u}^{*}\right)}$ and $\mathbf{A}_{2}=\frac{\partial \mathbf{f}}{\partial \hat{u}} \frac{\partial \hat{u}}{\partial u} \frac{\partial u}{\partial \mathbf{x}}$, but

$$
\mathbf{A}_{2}=\frac{1}{N+1} \frac{\partial \mathbf{f}}{\partial \hat{u}} \frac{\partial u}{\partial \mathbf{x}},
$$

and

$$
\mathbf{J}_{n}=\mathbf{A}_{1}+\frac{1}{N+1} \mathbf{A}_{u}
$$

Now, using the theorem of continuity of the eigenvalues, we get that if $N$ is sufficiently high, the eigenvalues of matrix $\mathbf{J}_{n}$ approximate the eigenvalues of matrix $\mathbf{J}_{o}$, and since all of them are inside the unit circle, system (4) is stable. $\triangleright$

Let us specify this result to systems operating with time-delayed signals.

Consequence 2: In the same hypotheses as in Result 1, the control design (FPIC technique)

$$
\hat{u}(\mathbf{x}(k))=\frac{u(\mathbf{x}(k-1))+N u^{*}}{N+1}
$$

stabilises the fixed point $\left(\mathbf{x}^{*}, u^{*}\right)$ for $N$ sufficiently high.

Note: The new signal control generated in this way does not move the fixed point. However, it is well-known that systems tend to destabilize when delay periods are considered.

\section{Proof:}

Considering one delay period, the system equations read as

$$
\mathbf{x}(k+1)=\mathbf{f}(\mathbf{x}(k), u(\mathbf{x}(k-1))) .
$$

The change of variables $\mathbf{z}_{1}=\mathbf{x}(k)$ and $\mathbf{z}_{2}=\mathbf{x}(k-$ 1), yields to

$$
\begin{aligned}
& \mathbf{z}_{1}(k+1)=\mathbf{f}\left(\mathbf{z}_{1}(k), u\left(\mathbf{z}_{2}(k)\right)\right) \\
& \mathbf{z}_{2}(k+1)=\mathbf{z}_{1}(k) .
\end{aligned}
$$

Then, with the new control signal

$$
\hat{u}(k)=\frac{u(\mathbf{x}(k-1))+N u^{*}}{N+1}
$$

state equations can be written as:

$$
\begin{aligned}
& \mathbf{z}_{1}(k+1)=\mathbf{f}\left(\mathbf{z}_{1}(k), \frac{u\left(\mathbf{z}_{2}(k)\right)+N u^{*}}{N+1}\right) \\
& \mathbf{z}_{2}(k+1)=\mathbf{z}_{1}(k)
\end{aligned}
$$

In this case, matrix $\mathbf{A}$, valued at the fixed point, can be computed as

$$
\mathbf{A}=\left.\left[\begin{array}{cc}
\frac{\partial \mathbf{f}}{\partial \mathbf{z}_{1}} & \frac{1}{N+1} \frac{\partial u}{\partial \mathbf{z}_{2}} \\
\mathbf{I} & \mathbf{0}
\end{array}\right]\right|_{\left(\mathbf{z}_{1}^{*}, \mathbf{z}_{2}^{*}\right)}
$$

being $\mathbf{I}$ the identity matrix and $\mathbf{0}$ a null matrix of appropriate dimensions. Then,

$$
\mathbf{A}=\left.\left[\begin{array}{cc}
\frac{\partial \mathbf{f}}{\partial \mathbf{z}_{1}} & \mathbf{0} \\
\mathbf{I} & \mathbf{0}
\end{array}\right]\right|_{\left(\mathbf{z}_{1}^{*}, \mathbf{z}_{2}^{*}\right)}+\left.\left[\begin{array}{cc}
\mathbf{0} & \frac{1}{N+1} \frac{\partial u}{\partial \mathbf{z}_{2}} \\
\mathbf{0} & \mathbf{0}
\end{array}\right]\right|_{\left(\mathbf{z}_{1}^{*}, \mathbf{z}_{2}^{*}\right)}
$$

In a simplified form $\mathbf{A}=\mathbf{A}_{1}+\mathbf{A}_{2}$ where $\mathbf{A}_{1}$ corresponds to the first matrix and $\mathbf{A}_{2}$ to the second one in (12). The eigenvalues of $\mathbf{A}_{1}$ are the eigenvalues of equation (2) plus $n$ eigenvalues at the origin. Applying the theorem of continuity of the eigenvalues for $N$ sufficiently high, the eigenvalues of $\mathbf{A}$ are close to the eigenvalues of $\mathbf{A}_{1}$, and since all of them are in the unit circle, the new controlled system is stable. $\triangleright$

\section{CONTROL OF CHAOS IN A CPWM BUCK CONVERTER}

This section starts describing the plant, a ZAD buck converter. Then, a Time-Delay AutoSynchronization (TDAS) as well as Fixed Point Inductor Control (FPIC) schemes are used to stabilise 1-periodic orbits close to the chaotic attractor.

\subsection{The buck converter}

Fig. 2 shows a schematic diagram of a buck converter which can be modelled as the dynamical system

$$
\left(\begin{array}{c}
\dot{v_{C}} \\
{\dot{i_{L}}}^{\prime}
\end{array}\right)=\left(\begin{array}{cc}
-\frac{1}{R C} & \frac{1}{C} \\
-\frac{1}{L} & 0
\end{array}\right)\left(\begin{array}{l}
v_{C} \\
i_{L}
\end{array}\right)+\left(\begin{array}{c}
0 \\
\frac{E}{L}
\end{array}\right) u
$$

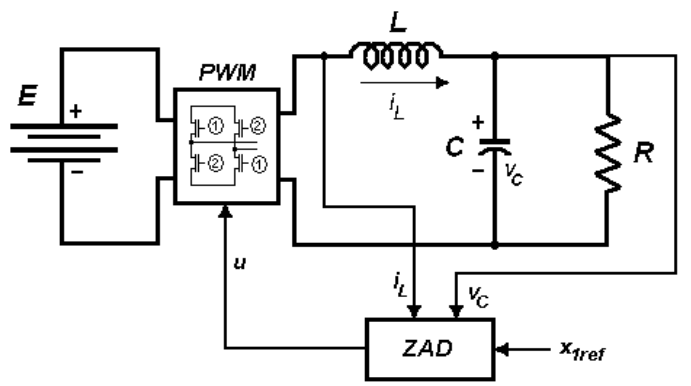

Fig. 2. Scheme of the blocks diagram of a PWM driving $\mathrm{DC} / \mathrm{AC}$ converter.

The capacitor voltage $v_{C}$ and the inductor current $i_{L}$ are the state variables. The control signal $u$ takes discrete values $\{-1,1\}$ depending on the switch position. The converter can also be described by the dimensionless system

$$
\left(\begin{array}{c}
\dot{x}_{1} \\
\dot{x}_{2}
\end{array}\right)=\left(\begin{array}{ll}
-\gamma & 1 \\
-1 & 0
\end{array}\right)\left(\begin{array}{l}
x_{1} \\
x_{2}
\end{array}\right)+\left(\begin{array}{l}
0 \\
1
\end{array}\right) u
$$


with $x_{1}=v / E, x_{2}=\frac{1}{E} \sqrt{\frac{L}{C}} i, t=\tau / \sqrt{L C}$ and $\gamma=\frac{1}{R} \sqrt{\frac{L}{C}}$ (Fossas and Zinober, 2001).

The system is controlled by PWM forcing zero average in the output $y=s(x)$ for each sampling period. For robustness purposes output $s$, a proportional-derivative expression in the error, as in (Carpita et al., 1988), is defined by

$$
s(x)=\left(x_{1}-x_{1 r e f}\right)+k_{s}\left(\dot{x}_{1}-\dot{x}_{1 \text { ref }}\right)
$$

where $x_{1}$ is the controlled variable, $x_{1 \text { ref }}$ is the reference signal and $k_{s}$ is the surface time constant. The control signal provided to the system is defined as follows

$u=\left\{\begin{array}{cl}1 & \text { if } k T \leq t \leq k T+d / 2 \\ -1 & \text { if } k T+d / 2<t<k T+(T-d / 2) \\ 1 & \text { if } k T+(T-d / 2) \leq t<(k+1) T\end{array}\right.$

where $d$ is computed by

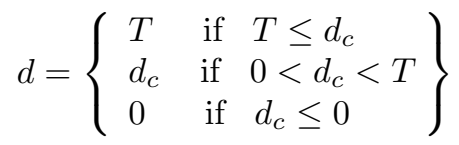

and

$$
d_{c}=\frac{2 s(k T)+T \dot{s}_{2}(k T)}{\dot{s}_{2}(k T)-\dot{s}_{1}(k T)} .
$$

In Eq. (18) $s(k T)$ corresponds to the surface value when states are evaluated at $t=k T . \dot{s}_{1}(k T)$ is the surface slope with control signal $u=1$ and $\dot{s}_{2}(k T)$ is the surface slope with $u=-1$. More details abot this strategy can be found in (Fossas et al., 2001).

\subsection{TDAS Control of a CPWM Buck Converter}

The goal of this technique is to feedback a timedelayed variable (Pyragas, 1992). Since the variable inducing instability can be associated to the duty cycle, we have

$$
d(k)=d+\eta(d(k)-d(k-1))
$$

where $d(k)$ is the duty cycle to be applied, $d$ is defined in the previous subsection and $d(k-1)$ is the duty cycle in the latest iteration ( $\eta$ is the feedback constant).

Figure 3 shows the duty cycle evolution when $k_{s}=0.5$. The dispersed points corresponds to the chaotic behavior when the system is not controlled through TDAS. The continuous line corresponds to the duty cycle when the system is TDAScontrolled. The values of the state variables in the stroboscopic Poincare map, for the stationary point are $x_{1}=0.8010$ and $x_{2}=0.2804$, with a regulation error of $0.125 \%$.

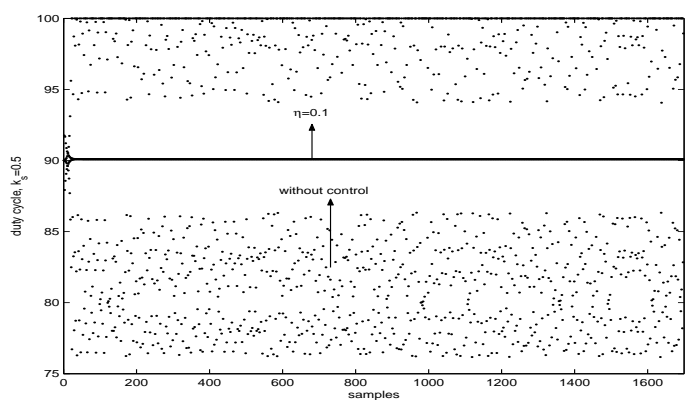

Fig. 3. Duty cycle for the uncontrolled system and with TDAS control. Points correspond to the uncontrolled system while the continuous line corresponds to the TDAS-controlled system, $k_{s}=0.5$.

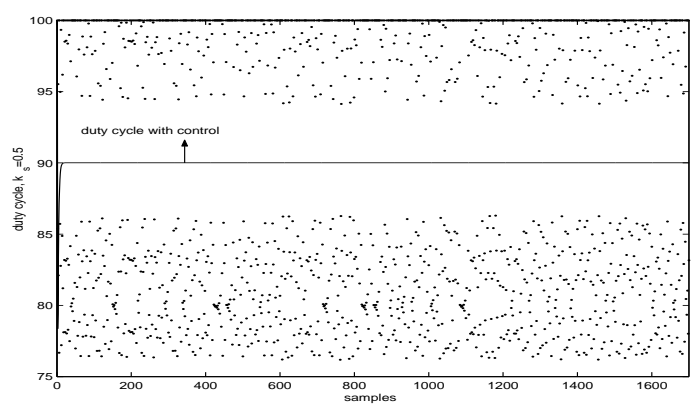

Fig. 4. Duty cycle for the uncontrolled system and for the FPIC-controlled system, $k_{s}=0.5$.

\subsection{FPIC Control of the CPWM Buck Converter}

The duty cycle in the steady-state can be easily computed, and it is found to be

$$
d_{s s}=T \frac{1+x_{1 r e f}}{2} .
$$

This allows applying the FPIC technique discussed in the previous Section. Now, the duty cycle to control the PWM is

$$
d(k)=\frac{d+N d_{s s}}{N+1}
$$

where $d$ is defined in equation (17) and $N \geq 1$ for the parameter range used. Fig. 4 shows the results when the system is controlled in this way. These results were obtained for $k_{s}=0.5$ and $N=1$, operating in the chaotic zone. The steadystate values are $x_{1}=0.7999$ and $x_{2}=0.2801$, with an error of $0.0125 \%$. Moreover, it is worth to note that the unstable orbit achieves the stable 1-periodic behavior as fast as using the TDAS technique. FPIC technique also allows stabilising higher periods orbits. In (Angulo et al., 2005) it was shown that when the stability of the 1periodic orbit is lost, through a flip bifurcation, a stable 2-periodic orbit appears together with a 1-periodic unstable orbit.

In order to get some indications of the robustness of the method, Fig. 5 shows the behavior of $x_{1}$ when sudden changes in the load are simulated. We have computed open-circuit events, which is 


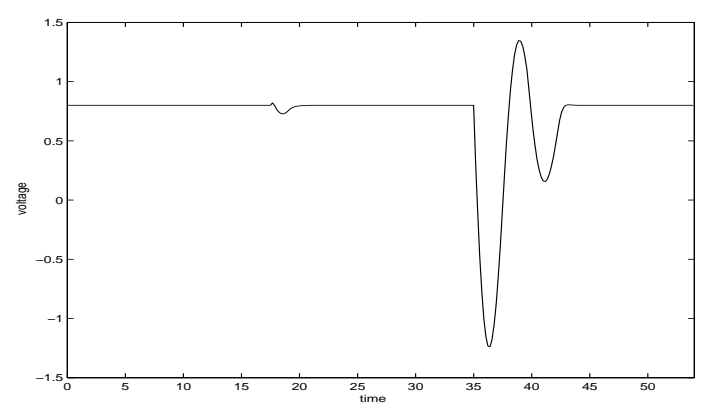

Fig. 5. Voltage behavior for the CPWM with FPIC. The values of the parameters are $k_{s}=$ 0.5 and $N=1$. For $t=18 s$ nominal control operation is simulated. For $t=36 \mathrm{~s}$ we simulate a change from nominal control to $10 \%$ of the load nominal value.

equivalent to assume $\gamma=0$, and a resistance drop to a $10 \%$ of the nominal value of the load with $\gamma=3.5$. This sort of phenomena usually happens in power systems. The open-circuit operation is simulated for $t=18 \mathrm{~s}$. The controller response takes no more than 20 sampling periods. This is equivalent to stabilize the system in $1 \mathrm{~ms}$. When $t=36 \mathrm{~s}$, the system changes from open-circuit operation to $10 \%$ of the load nominal value. In this case, the stabilization time is $2.5 \mathrm{~ms}$ approximately (50 sampling periods). We assumed that initially the system was operating in the chaotic region, with $k_{s}=0.5$, and stabilises it with an FPICcontroller taking $N=1$. As a conclusion we set that the trade off the FPIC holds between the unstabilising control and a nominal control action, keeps the robustness of the ZAD controller.

\section{CONTROL OF A DCPWM BUCK CONVERTER}

We will show now some results concerning a Delayed Centered PWM Buck Converter (DCPWM). These results concern only the Regulation problem although other simulations not included in this communication show also very good results for the Tracking problem.

In this case, since a one period delay is considered, the fixed point is not moved. Thus the applied duty cycle $d(k)$ has the same expression that in the previous case,

$$
d(k)=\frac{d+N d_{s s}}{N+1}
$$

where $d$ is evaluated as in (17), at $(k-1) T$, namely

$$
d_{c}=\frac{2 s(\mathbf{x}((k-1) T))+T \dot{s}_{2}(\mathbf{x}((k-1) T))}{\dot{s}_{2}(\mathbf{x}((k-1) T))-\dot{s}_{1}(\mathbf{x}((k-1) T))}
$$

This expression for $d_{c}$ is different from the CPWM case and yields to an unstable equilibrium point. Fig. 1 shows a bifurcation diagram when this

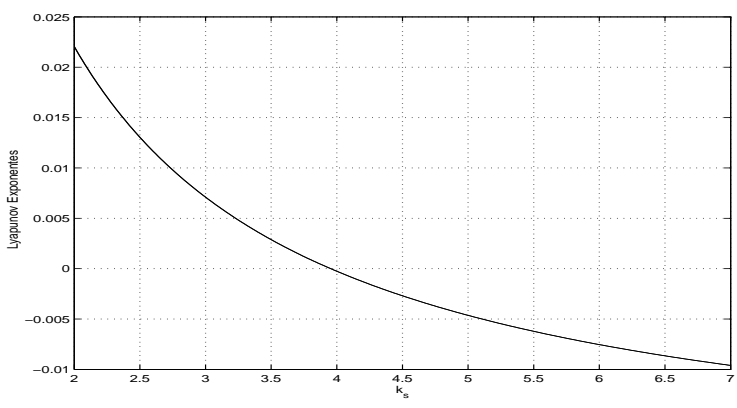

(a)

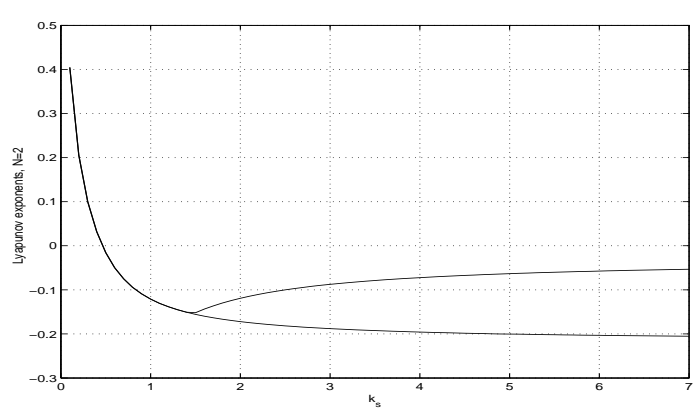

(b)

Fig. 6. Lyapunov exponents for the FPICcontrolled DCPWM, (a) with $N=1$; (b) with $N=2$.

duty cycle is applied. The Lyapunov exponents of the controlled system (for $N=1$ ) are shown in Fig. 6 (a). It can be observed that the 1-periodic orbit has changed, from being unstable for all $k_{s}$ to being stable for $k_{s}>3.9$. If we want to widen the stability range, one can increase $N$. Fig. 6 (b) shows the evolution of the Lyapunov exponents when $N=2$. The stability limit drops drastically and is located near 0.5 . This sudden change between $N$ and $N+1$ allows taking small values for $N$ generally.

\section{CONCLUSIONS}

In this paper we have shown the usefulness of a novel and simple stabilising technique for unstable and chaotic systems. Only analytical or numerical values of a steady-state control input guaranteeing a stable equilibrium point are needed. This technique has been checked to be efficient for a PWM-controlled buck converter with ZAD strategy. It has also been checked for a CPWM with 1-period delay and ZAD strategy, with very good results. The controller stability is guaranteed by computing the Lyapunov exponents.

Simulations also show that the trade off the FPIC holds between the unstabilising control and a 
nominal control action, keeps the properties of the closed-loop controller.

The behavior of the dynamics in the PWMcontrolled buck converter with ZAD strategy was compared using TDAS and FPIC techniques. The latter shows better response in robustness, convergence velocity, less error in the steady state, with the important advantage that it does not require state extra-measurements.

The design value for $N$, analytically, is currently the objective of further work.

\section{REFERENCES}

Angulo, F., E. Fossas and G. Olivar (2005). Transition from periodicity to chaos in a pwmcontrolled buck converter with zad strategy. International Journal of Bifurcations and Chaos, accepted.

Batlle, C., E. Fossas and G. Olivar (1997). Time delay stabilization of the buck converter. In Proceedings of the IEEE International Conference in Control of Oscillations and Chaos 3, 590-593.

Batlle, C., E. Fossas and G. Olivar (1999). Stabilization of periodic orbits of the buck converter by time-delayed feedback. International Journal of Circuit Theory and Applications 27, 617-631.

Batlle, C., E. Fossas and G. Olivar (2000). From floquet exponents to control of chaos in piecewise-linear systems. In Proceedings of the IEEE International Symposium on Circuits and Systems, Geneva, Switzerland pp. 100-103.

Biel, D., E. Fossas, R. Ramos and F. Guinjoan (2002). Implementación de controles "pseudosliding" en sistemas conmutados (in spanish). Congreso Latinoamericano de Control Automático, Guadalajara, México.

Borle, L.J. and N.V. Chemmangot (1995). Zero average current error controlled power flow for ac-dc power converters. IEEE Transactions on Power Electronics 10, No. 6, 725732.

Carpita, M., M. Marchesoni, M. Oberti and L. Puguisi (1988). Power conditioning system using slide mode control. In Proceedings of the IEEE Power Electronics Specialist Conference pp. 623-633.

Fossas, E. and A. Zinober (2001). Adaptive tracking control of nonlinear power converters. In Proceedings of the IFAC Workshop on Adaptation in Control and Signal Processing, Connobio, Italy pp. 264-266.

Fossas, E., R. Griñó and D. Biel (2001). Quasisliding control based on pulse width modulation, zero average and the $l_{2}$ norm. In $A d$ - vances in Variable Structure System, Analysis, Integration and Applications pp. 335-344.

Malesani, L., L. Rosetto, G. Spiazzi and A. Zucatto (1996). An ac power supply with sliding mode control. IEEE Industry Applications Magazine pp. 32-38.

Nicolas, B., M. Fadel and Y. Chron (1996). Fixed frequency sliding mode control of a singlephase voltage source inverter with input filter. In Proceedings of the IEEE International Symposium on Industrial Electronics 1, 470475.

Pinheiro, H., A.S. Martins and J.R. Pinheiro (1994). A sliding mode controller in single phase voltage source inverters. In Proceedings of the IEEE International Conference on Industrial Electronics, Control and Instrumentation pp. 394-398.

Poddar, G., K. Chakrabarty and S. Banerjee (1995a). Control of chaos in the boost converter. Electronics letters 31, No. 11, 841842.

Poddar, G., K. Chakrabarty and S. Banerjee (1995b). Experimental control of chaotic behavior of buck converter. IEEE Transcations on Circuits and Systems-I: Fundamental Theory and Applications 42, No. 8, 502-504.

Poddar, G., K. Chakrabarty and S. Banerjee (1998). Control of chaos in dc-dc converters. IEEE Transcations on Circuits and SystemsI: Fundamental Theory and Applications 45, No. 6, 672-676.

Pyragas, K. (1992). Continuous control of chaos by self-controlling feedback. Physics Letters A 170, 421-428.

Ramos, R., D. Biel, E. Fossas and F. Guinjoan (2003). A fixed-frequency quasi-sliding control algorithm: Application to power inverters design by means of fpga implementation. IEEE Transcations on Power Electronics.

Ruiz, J.M., S. Lorenzo, I. Lobo and J. Amigo (1990). Minimal ups structure with sliding mode control and adaptative hysteresis band. In Proceedings of the IEEE International Conference on Industrial Electronics, Control and Instrumentation 2, 1063-1067.

Silva, J.F. and S.P. Snia (1993). Fixed frequency sliding mode modulator for current mode pwm inverters. In Proceedings of the IEEE Power Electronics Specialists Conference pp. 623-629.

Yao, Q. and D.G. Holmes (1993). A simple, novel method for variable-hysteresis band current control of a three phase inverter with constant switching frequency. In Proceedings of the IAS Meeting pp. 1122-1129. 